\title{
Interdisciplinary Chemical Approaches for Neuropathology
}

\author{
Rona R. Ramsay ${ }^{1}$ \& Giuseppe Di Giovanni ${ }^{2,3}$ \\ 1 Biomedical Sciences Research Centre, University of St Andrews, St Andrews, UK \\ 2 Department of Physiology and Biochemistry, University of Malta, Msida, MSD 2080, Malta \\ 3 School of Bioscience, Cardiff University, Cardiff, UK
}

\author{
Correspondence \\ G. Di Giovanni, Department of Physiology and \\ Biochemistry, \\ University of Malta, \\ Msida MSD 2080, Malta. \\ Tel.: +356 23402776; \\ Fax: +356 21310577; \\ E-mail: giuseppe.digiovanni@um.edu.mt \\ and \\ Dr. Rona R. Ramsay, Biomedical Sciences \\ Research Complex, University of St Andrews, \\ Biomolecular Sciences Building, North Haugh, \\ St Andrews, KY16 9ST, UK. \\ Tel.: +44-(0)1334-463411; \\ Fax: +44-(0)1334-462595; \\ E-mail: rrr@st-andrews.ac.uk
}

doi: $10.1111 /$ cns. 12297

Brain monoamine neurotransmitters, which include dopamine (DA), noradrenaline (NA), adrenaline (A) serotonin (5-HT) histamine, and melatonin, play a central role in the pathophysiology of all major neuropsychiatric disorders, such as mood disorders, schizophrenia, autism-spectrum disorders, Parkinson disease (PD), epilepsy, and dementias [1]. Ligands for the receptors acted upon by the monoamine neurotransmitters and inhibitors of the enzymes for breakdown, and the reuptake transporters have been developed as drugs [2-4], but successful therapy of neuropsychiatric disorders is limited by the high variability of the symptoms and of the responses to treatment. Few drugs are available to address specific subsets of neurological/mental symptoms and none to aid in diagnosis or to stop the progress of neurodegenerative disorders.

Medicinal chemists now have many tools to aid design of novel drugs, but biological insight is essential. Both what to design and the accurate biological assessment of new compounds in vitro and in vivo are key to advances in treatment. With this in mind, a collaborative network of researchers began working toward a common goal from different perspectives in 2011. COST Action CM1103 (http://www.cost.eu/domains_actions/cmst/Actions/ CM1103) Structure-Based Drug Design For Diagnosis And Treatment of Neurological Diseases: Dissecting and Modulating Complex Function in the Monoaminergic Systems of the Brain was established to stimulate an interdisciplinary approach to the task of understanding the molecular basis of behavior in degeneration. The collaboration facilitates chemical biology by close and ongoing contacts between synthetic and computational chemists and molecular and physiological biologists. This collaboration has provided a depth of insight and will increase the probability of practical medicinal chemistry outcomes in terms of tools for neurochemistry and drugs for psychiatry. The articles in this special issue are a selection from the multidisciplinary contributions to the second annual meeting of the 30 groups actively working towards these goals.

The focus of the action is to design a series of multitarget compounds with action on specific pairs (or multiples) of targets. Multitarget drugs are an accepted strategy in tackling the complex neurodegenerative diseases [4-7], but producing the best combinations to explore phenotypic results is not a trivial task (Figure 1). With large numbers of compounds tested and published in databases, one starting point comes from data mining existing knowledge. Starting from known chemicals, theoretic prediction of pharmaceutical targets for new compounds is possible using a probabilistic method to build a model of any compound from the ChEMBL dataset. Each molecule is reduced to circular fingerprint descriptors. Application of the developed "predictor" software then takes the model and searches for primary pharmaceutical targets and off-targets [8]. Identified compounds are then studied experimentally (see the articles by Di Giovanni et al., Bolea et al., and Nikolic et al.).

The traditional approach starting from the targets makes use of the many crystal structures now available. Structures for the 


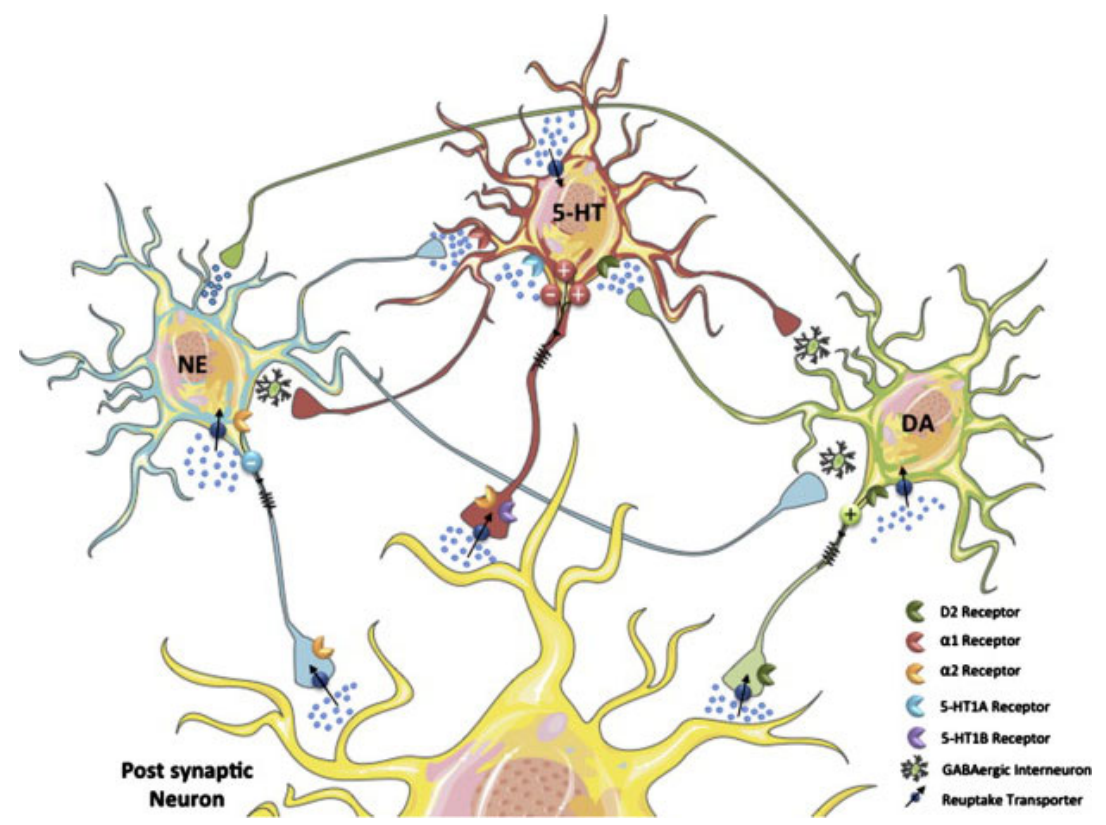

Figure 1 Functional connectivity of monoaminergic neurons. Direct and indirect (through GABAergic interneurons) interconnections between 5-HT, NE, and DA neurons are mediated through various receptor types which act both as autoreceptors controlling the activity of respective neuronal phenotype and heteroreceptors controlling the activity of the other two monoaminergic neuronal phenotypes. Combined pharmacological actions at reuptake transporters and selected auto/hetero-receptors allow finely controlled modulations of monoaminergic neurons reciprocal interconnections and impinging on postsynaptic neurons in brain circuits involved in neuropsychiatric disorders. Multitargeted drugs acting at both reuptake transporters and selected monoamine receptors are especially promising as more effective and better tolerated drugs. Reproduced with permission from [7].

enzymes that degrade the monoamine neurotransmitters (MAO and COMT) are available for computational exploration of molecular determinants of binding and even support thermodynamic analysis of putative mechanisms. Inhibition of monoamine oxidases (MAO A and MAO B) by several irreversible inhibitors and a few new, well-tolerated, reversible inhibitors used over the last 30 years results in increased levels of brain amines [9]. Docking and molecular dynamic studies of the inhibitor in the active site are now standard tools for medicinal chemists aiming to improve inhibitor binding or decide which part of a molecule may be changed without loss of affinity. This approach has facilitated the addition of activities to the rasagiline scaffold [10] and a new combined cholinesterase and monoamine oxidase inhibitor [11]. Using structure-based techniques, complete theoretic searches for new lead compounds are also possible [12].

Reuptake transporters are also targets to modulate amine levels in the synaptic cleft; see for example [13]. In the absence of structures, homology models of the transporters in conjunction with mutational studies are beginning to define the molecular determinants of binding to these proteins. Structure-based drug design together with good pharmacological data provides the basis for designs combining the features needed for each target into one molecule.

The action of monoamines is effected via specific families of receptors which serve different but complementary roles in the control of cognitive functions and behavior [14]. The receptors are G Protein-Coupled Receptors (GPCRs), a large class of proteins responsible for transferring chemical signals from the exterior of a cell to its interior. Recent advances in structural biology have enabled the crystallization of a very small number of GPCR proteins, for example, the structure of the human D3 receptor in complex with a D2/D3 selective antagonist. This field is developing so rapidly that a new collaborative action has begun to design drugs and chemical tools for these targets: GLISTEN: GPCR-Ligand Interactions, Structures, and Transmembrane Signalling: a European Research Network (http://www.cost.eu/domains_actions/ cmst/Actions/CM1207).

The pharmacological modulation of the signalling of select monoamine receptors is a key therapeutic strategy to attenuate the severity of numerous mental disorders. For example, the antagonism of D2 (and perhaps also D3 and D4) DA receptors and $5-\mathrm{HT}_{2 \mathrm{~A}}$ serotonin receptors is fundamental to reduce the severity of psychotic symptoms [15]. In this issue, new evidence is shown about role of the $5-\mathrm{HT}_{2 \mathrm{C}}$ and somatostatin receptors in temporal lobe epilepsy by the Di Giovanni and Smolders groups, respectively. Navailles et al. showed that the participation of NA transporters in the clearance of DA may account for the lower effect of L-DOPA in extrastriatal regions compared with the striatum. In addition, Navailles, Di Giovanni and De Deurwaerdère review available data to address the extent to which 5-HT electrical activity contributes to the effect of L-DOPA on both DA and 5-HT releases in PD. Naumenko and co-workers review the possible cross-talk between two serotonin receptors, $5-\mathrm{HT}_{1 \mathrm{~A}}$ and the $5-\mathrm{HT}_{7}$, and its role in different neuropsychiatric disorders. Frau et al. present data supporting the implication of $\mathrm{GABA}_{\mathrm{B}}$ receptors 
in the modulation of sensorimotor gating and point to their positive allosteric modulators as a novel promising tool for antipsychotic treatment, with fewer side effects than $\mathrm{GABA}_{\mathrm{B}}$ receptor agonists. In addition, substantial progress has been made toward understanding the genetic architecture, cellular substrates, brain circuits, and endophenotypic profiles of neuropsychiatric disorders (see in this issue, Cauchi, Camilleri et al., Mifsud et al., Babic et al., Fogel et al.).

Whatever the approach used to identify interesting new compounds, the biological evaluation remains the key. The ligand affinities for all compounds against all receptors, transporters, and enzymes must be determined experimentally to support the computational assessments and further optimization. Such work is generally high throughput now, providing the target is validated, and efficient assays have been developed. But what is the target in the highly variable presentations of neurodegenerative diseases? For neurodegeneration, where multitarget drugs are the trend, biological assessment means sending the compounds to a multitude of different labs each with expertise in subsets of the targets.

Even with a rich armory of chemical approaches, cell- or animal-based phenotypic screening is still the vital test. Conversely, the chemicals are tools for pharmacological advances to understand the complex system of the brain. Chemicals designed to hit a variety of multiple targets in known proportions will allow studies of changes in signalling pathways, protein expression, and energy status of cells [7]. Such tools are necessary for the identification of new targets for validation. Certainly for $\mathrm{AD}$, the cause of the loss of neurons still awaits description, and causative rather than symptomatic targets in schizophrenia would also be an advance. The outcomes of a collaborative approach will be lead compounds for diagnosis and personalized treatment of complex neurological diseases. These are tools for future translational studies to elicit specific therapeutic and neurochemical effects in vivo. The articles in this special are small steps along that path.

\section{Acknowledgments}

The collaboration among the researchers that contributed to this special issue was funded by EU COST Action CM1103 “Structurebased drug design for diagnosis and treatment of neurological diseases: dissecting and modulating complex function in the monoaminergic systems of the brain". The articles included in this special issue were presented at the CM1103 Action annual conference "Interdisciplinary Chemical Approaches for Neuropathology" joined to “4th Neuroscience Day@ University of Malta”, organized by Prof. Di Giovanni as President of Malta Physiological Society (MPS) in Malta, October 2013 (http://www.um.edu.mt/events/ neuropathology2013). The conference was supported by COST (European Cooperation in Science and Technology), MCST (Malta Council of Science and Technology) and Malta Chamber of Scientists. Our most hearty thanks go to Mr Buddy Zhou of Wiley, and Professor Ding-Feng Su, Editor of CNS N\&T, and the reviewers and all who contributed in one way or other in publishing this special issue.

\section{References}

1. Di Giovanni G, Di Matteo V, Esposito E. Serotonin-Dopamine Interaction: Experimental Evidence and Therapeutic Relevance. Amsterdam: Elsevier, 2008.

2. Ramsay RR. Monoamine oxidases: the biochemistry of the proteins as targets in medicinal chemistry and drug discovery. Curr Top Med Chem 2012;12:2189-2209.

3. Geldenhuys WJ, Youdim MBH, Carroll RT, Van der Schyl CJ. The emergence of designed multiple ligands for neurodegenerative disorders. Prog Neurobiol 2011;94:347359

4. Van der Schyf CJ, Geldenhuys WJ, Youdim MBH. Multifunctional drugs with different CNS targets for neuropsychiatric disorders. J Neurochem 2006;99:10331048.

5. Kell DB. Finding novel pharmaceuticals in the systems biology era using multiple effective drug targets, phenotypic screening and knowledge of transporters: where drug discovery went wrong and how to fix it. FEBS J 2013;280:5957-5980.
6. Hamann J, Rommelspacher H, Storch A, Reichmann H, Gille G. Neurotoxic mechanisms of 2,9-dimethyl-beta-carbolinium ion in primary dopaminergic culture. J Neurochem 2006;98:1185-1199.

7. Hamon M, Blier P. Monoamine neurocircuitry in depression and strategies for new treatments. Prog Neuropsychopharmacol Biol Psychiatry 2013;45:54-63.

8. Koutsoukas A, Lowe R, KalantarMotamedi Y, et al. In silico target predictions: defining a benchmarking data set and comparison of performance of the multiclass naive bayes and parzen-rosenblatt window. J Chem Inf Model 2013;53:1957-1966.

9. Youdim MB, Bakhle YS. Monoamine oxidase: isoforms and inhibitors in Parkinson's disease and depressive illness. Br J Pharmacol 2006;147(Suppl 1):S287-S296.

10. Youdim MBH. Multi target neuroprotective and neurorestorative anti-Parkinson and anti-Alzheimer drugs ladostigil and $\mathrm{m} 30$ derived from rasagiline. Exp Neurobiol 2013;22:1-10.

11. Samadi A, de los Rios C, Bolea I, et al. Multipotent MAO and cholinesterase inhibitors for the treatment of
Alzheimer's disease: synthesis, pharmacological analysis and molecular modeling of heterocyclic substituted alkyl and cycloalkyl propargyl amine. Eur J Med Chem 2012;52:251-262.

12. Vilar S, Ferino G, Quezada E, Santana L, Friedman C. Predicting monoamine oxidase inhibitory activity through ligand-based models. Curr Top Med Chem 2012;12:22582274

13. Carter DS, Cai H-Y, Lee EK, et al. 2-Substituted N-aryl piperazines as novel triple reuptake inhibitors for the treatment of depression. Bioorg Med Chem Lett 2010;20:3941-3945.

14. Di Giovanni G. New ligands at 5-HT and DA receptors for the treatment of neuropsychiatric disorders. Curr Top Med Chem 2008;8:1005-1007

15. Wood MD, Wren PB. Serotonin-dopamine interactions: implications for the design of novel therapeutic agents for psychiatric disorders. In: Di Giovanni G, Di Matteo V, Esposito E, editors. Serotonin-Dopamine Interaction: Experimental Evidence and Therapeutic Relevance. Amsterdam: Elsevier, 2008;213-230. 